\title{
Beam dynamics design of a new radio frequency quadrupole for beam-current upgrade of the Japan Proton Accelerator Research Complex linac
}

\author{
Yasuhiro Kondo, ${ }^{*}$ Kazuo Hasegawa, and Takatoshi Morishita \\ Japan Atomic Energy Agency (JAEA), Tokai, Naka, Ibaraki, 319-1195, Japan \\ Robert A. Jameson \\ Institüt Angewandte Physik, Goethe-Universität Frankfurt, Frankfurt am Main D60438, Germany
}

(Received 5 July 2012; published 23 August 2012)

\begin{abstract}
We have performed the beam-dynamics design of an $\mathrm{H}^{-}$radio frequency quadrupole (RFQ) for the beam-current upgrade of the Japan Proton Accelerator Research Complex linac (RFQ III). LINACSrfqDES was set up to support the conventional design method, i.e., design with CURLI-type shaper followed by constant bore radius and with constant intervane voltage, supplemented by keeping the equipartitioned condition in the gentle buncher section. For the particle simulation, LINACSrfqSIM was used. The obtained transmission, transverse and longitudinal emittance are 98.5\%, $0.21 \pi \mathrm{mm} \mathrm{mrad}$, and $0.11 \pi \mathrm{MeV}$ deg for the input beam current of $60 \mathrm{~mA}$ and normalized rms emittance of $0.20 \pi \mathrm{mm}$ mrad. This design satisfies the requirements of RFQ III.
\end{abstract}

DOI: 10.1103/PhysRevSTAB.15.080101

PACS numbers: 29.20.Ej, 29.27.Bd, 29.27.Fh

\section{INTRODUCTION}

Japan Proton Accelerator Research Complex (J-PARC) is a multipurpose facility for particle physics, nuclear physics, materials and life science, and so on. The J-PARC accelerator consists of a $400 \mathrm{MeV}$ linac, a $3 \mathrm{GeV}$ rapid-cycling synchrotron [1], and a $50 \mathrm{GeV}$ main ring [2]. The original design energy and peak beam current of the linac is $400 \mathrm{MeV}$ and $50 \mathrm{~mA}$, though, at the initial phase, the operation was started with an energy of $181 \mathrm{MeV}$ omitting annular-coupling structures [3]. A radio frequency quadrupole (RFQ) built for the Japan hadron facility project is used for the initial-phase linac; the design peak beam current of this RFQ is $30 \mathrm{~mA}$ [4]. To achieve the original design power of $1 \mathrm{MW}$ (at the neutron target), it is planned to upgrade the linac to the original $400 \mathrm{MeV}$ and $50 \mathrm{~mA}$ [1]. To upgrade the beam current, an RFQ with a design current of $50 \mathrm{~mA}$ is newly fabricated and will replace the old one. This 50-mA RFQ is called RFQ III [5]. In Table I, requirements for J-PARC RFQ III are listed.

For designing RFQs, a method proposed by Los Alamos National Laboratory [8] is one of the most popular design methods. In this method, an RFQ is divided into four sections: a radial matching section (RMS), a shaper (SP), a gentle buncher (GB), and an accelerator (ACC). Additionally, intervane voltage $V$ and average bore radius $r_{0}$ are kept constant except for the RMS; this means focusing strength $B$ is kept constant. In this conventional

\footnotetext{
*yasuhiro.kondo@j-parc.jp
}

Published by the American Physical Society under the terms of the Creative Commons Attribution 3.0 License. Further distribution of this work must maintain attribution to the author(s) and the published article's title, journal citation, and DOI. method, the transverse focusing force remains strong even in the ACC, where the space-charge force is relatively weak and the transverse focusing force need not be so strong. This causes insufficient longitudinal force and ineffective acceleration efficiency. Therefore, in these days, some RFQs are designed to reduce $B$ (increase $r_{0}$ ) and make $V$ higher with progress of acceleration to obtain more acceleration efficiency $[9,10]$.

On the other hand, the conventional method still has merits in fabricating RFQ cavities. First of all, if $r_{0}$ and $V$ are constant, the resonant frequency of the cross-sectional shape can be constant, and therefore, frequency tuning using vane-skirt shape or cavity radius is not needed. Thus, the longitudinally same cross-sectional shape makes it simple to design and machine the cavity. Second, it is possible to machine constant- $r_{0}$ and constant- $\rho_{t}$-ratio vanes using formed cutters. Even if ball-nose cutters are used, the computer-aided-machining program for constantcross-section vanes is very simple. Moreover, the field distribution is uniform along the RFQ and this makes it easier to tune the cavity in the fabrication process. Additionally, the stability during operation is expected to

TABLE I. Requirements for J-PARC RFQ III.

\begin{tabular}{lc}
\hline \hline Beam species & $\mathrm{H}^{-}$ \\
Resonant frequency & $324 \mathrm{MHz}$ \\
Injection energy & $50 \mathrm{keV}$ \\
Extraction energy & $3 \mathrm{MeV}$ \\
Peak beam current & $50 \mathrm{~mA}$ \\
Transverse normalized rms emittance & $<0.25 \pi \mathrm{mm} \mathrm{mrad}$ \\
Repetition rate & $50 \mathrm{~Hz}$ \\
rf pulse length & $600 \mu \mathrm{s}$ \\
rf duty factor & $3 \%$ \\
\hline \hline
\end{tabular}


be better because of the longitudinally uniform structure. Considering these merits and our experience of RFQ I and RFQ II, the conventional constant $-r_{0}$ and $-V$ design is adopted for J-PARC RFQ III.

In this paper, the beam-dynamics design of J-PARC RFQ III is described. The detail of design scheme is shown in Sec. II. In Sec. III, the particle simulation code used in the design process is presented and the results are shown in Sec. IV. Finally, in Sec. V, we summarize the paper.

\section{DESIGN}

For the beam-dynamics design of RFQ III, LINACSrfqDES $[11,12]$ was used. LINACSrfqDES is produced by one of the authors (R.A.J.), which is a MATHEMATICA [13] based quite flexible tool. A lot of design schemes are implemented in it, although the most essential design scheme is the equipartitioning. In this section, the original equipartitioning scheme is described first, then the modified point for the design of RFQ III is explained.

In the beam dynamics of LINACSrfqDES, the beam is required to be a matched beam in a focusing system. The matched beam is described using the smooth approximation as follows:

$$
\begin{gathered}
\varepsilon_{t n}=\frac{r^{2} \sigma_{t} \gamma}{\lambda}, \\
\varepsilon_{l n}=\frac{(\gamma b)^{2} \sigma_{l} \gamma}{\lambda},
\end{gathered}
$$

where $\varepsilon_{t n}$ and $\varepsilon_{l n}$ are the transverse and longitudinal normalized rms emittances, $r$ and $b$ are the transverse and longitudinal beam radii, $\lambda$ is the rf wavelength, and $\gamma$ is the relativistic gamma. The $\sigma_{t}$ and $\sigma_{l}$ are transverse and longitudinal phase advances; they are described with zero current phase advances $\sigma_{0}^{t}, \sigma_{0}^{l}$, and beam current $I$ as

$$
\begin{aligned}
& \varepsilon_{t n}^{2}=\frac{r^{4} \gamma^{2}}{\lambda^{2}}\left\{\sigma_{0}^{t 2}-\frac{I \lambda^{3} k(1-f)}{r^{2}(\gamma b) \gamma^{2}}\right\} \\
& \varepsilon_{l n}^{2}=\frac{(\gamma b)^{4} \gamma^{2}}{\lambda^{2}}\left\{\sigma_{0}^{l 2}-\frac{2 I \lambda^{3} k f}{r^{2}(\gamma b) \gamma^{2}}\right\} .
\end{aligned}
$$

The constant $k=\frac{3}{8 \pi} \frac{Z_{0} q \times 10^{-6}}{m_{0} c^{2}}$ is written with impedance of vacuum $Z_{0}$, the charge and the rest mass of the particle $q$ and $m_{0}$, the velocity of light $c$, and the ellipsoid form factor $f$ [14]. The zero current phase advances $\sigma_{0}^{t}$ and $\sigma_{0}^{l}$ are defined by the structural parameters of the focusing system as

$$
\begin{gathered}
\sigma_{0}^{t 2}=\frac{B}{8 \pi^{2}}+\Delta_{\mathrm{rf}}, \\
\sigma_{0}^{l 2}=2 \Delta_{\mathrm{rf}} .
\end{gathered}
$$

The focusing factor $B$ is

$$
B=\frac{q \lambda^{2} V}{m_{0} c^{2} r_{0}^{2}}
$$

and the rf defocusing factor $\Delta_{\mathrm{rf}}$ is [8]

$$
\Delta_{\mathrm{rf}}=\frac{\pi^{2} q V A \sin \phi_{s}}{2 m_{0} c^{2} \beta^{2} \gamma^{3}}
$$

where $\phi_{s}$ is the synchronous phase angle and $\beta$ is the relativistic beta. The acceleration parameter $A$ is described with multipole components of the external field as $A=$ $A_{10}+A_{30}$. The $A_{10}$ and $A_{30}$ are parametrized with the geometrical parameters of the vane tip, that is, the vane modulation factor $m$, the aperture radius $a$ described at the vane tip minimal, and the cell length $l_{c}$ [15].

One other optional condition that characterizes the beam dynamics in LINACSrfqDES is the equipartitioning condition, used to avoid the effect of parametric resonances in high-current linacs [16]. This condition requires the internal energy in the transverse and the longitudinal phase spaces of the beam to be the same, i.e.,

$$
\frac{\varepsilon_{l n} \sigma_{l}}{\varepsilon_{t n} \sigma_{t}}=1 \text {. }
$$

Where this condition is satisfied, there is no free energy to drive a resonance.

In the RFQ design scheme of LINACSrfqDES, the independent variables are $m, a, \phi_{s}$, and $V$, which may be used to solve for secondary variables such as the equipartitioning condition or constant $B$, etc. along the RFQ, or must be specified by rules as functions of cell number or beta or position, etc. For the equipartitioning condition, $m$ is found by solving the simultaneous equations (1), (2), and (9) for $r, b$, and $m$. The $\varepsilon_{t n}$ and $\varepsilon_{l n}$ are given parameters in the program.

The design scheme described above is the scheme of basic LINACSrfqDES, but it is easily modified for any desired external field or beam space-charge behavior. The most essential feature of LINACSrfqDES is the beambased design, i.e., the ability to design RFQs including the space-charge physics. The choices of quantities like $r_{0}$ and $V$ from the convenience of fabricating and tuning the cavities are the prerogative of the designer, and can be set up by user-defined rules. This flexibility to adapt various conditions is one of the features of LINACSrfqDES.

The design sequence is as follows. First of all, $a$ is derived from $m$ with the constant- $r_{0}$ condition. At a particular $z$ position ( $\sim$ cell 100$), m$ is calculated from the matching and equipartitioning conditions to achieve the equipartitioned condition, and special shaper parameters back to the end of the RMS are determined.

From this point, the governing rules are to keep the equipartitioned condition, while also keeping the transverse focusing $B$ and $r_{0}$ constant, and to govern the charge density in the bunch by giving a rule for the synchronous phase $\phi_{s}$, which is varied as 


$$
\begin{gathered}
\phi_{s}=\tan ^{-1}\left(\frac{\sin \Phi_{\mathrm{sep}}-\Phi_{\mathrm{sep}}}{1-\cos \Phi_{\mathrm{sep}}}\right), \\
\Phi_{\mathrm{sep}}=\frac{2 \pi \hat{b}}{\beta \lambda}, \\
\hat{b}=\left\{c_{1}+c_{2}\left(z-z_{\mathrm{EOS}}\right)\right\} b,
\end{gathered}
$$

where $c_{1}$ and $c_{2}$ are parameters to adjust the longitudinal acceptance, and $z_{\mathrm{EOS}}$ is $z$ at the end of SP. The vane voltage $V$ is constant, so $m$ and $a$ are used to satisfy the conditions.

However, as the accelerating force subtracts from the total available focusing force, the only way to keep $B$ and $r_{0}$ constant is to reduce the aperture $a$. At some value of the synchronous phase, $a$ will start to reduce drastically toward zero, and the procedure must be abruptly changed [17].

Therefore, after the transverse acceptance $A_{t}$ reaches a particular value, the scheme is changed to keep $A_{t}$ constant. This $z$ where $A_{t}$ reaches the particular value is so called the end of GB ( $\sim$ cell 238). If the transverse acceptance is kept, the longitudinal focusing force gets weaker; however, this would be acceptable because the bunching is essentially finished. The normalized transverse acceptance is defined as

$$
A_{t}=\frac{a^{2} \sqrt{\frac{B^{2}}{8 \pi^{2}}+\Delta_{\mathrm{rf}}+\Delta_{s c}}}{\beta \lambda\left(1+\frac{B}{4 \pi^{2}}\right)^{2}} \beta \gamma,
$$

where $\Delta_{s c}$ is the defocusing force by the space charge. The $A_{t}$ is a parameter decided by trading off the transmission and the vane length. We adopted the constant $-r_{0}$ and $-V$ design, so that this ACC does not meet the equiparitioning condition. However, the parameters quickly pass through the resonance regions, therefore, no significant emittance growth occurs. This point will be discussed later in Sec. IV.

In Table II, the design parameters of RFQ III obtained using the above scheme are summarized. The transverse cross section of the vane is a circular shape with radius $\rho_{t}$; the ratio of the $\rho_{t}$ to $r_{0}$ is constant $\left(\rho_{t} / r_{0}=0.75\right)$. The longitudinal vane shape is sinusoidal. This is because the acceleration factor of the sinusoidal vane is somewhat

TABLE II. Design parameters of J-PARC RFQ III obtained with LINACSrfqDES. In this table, TC and FFS mean transition cell [19] and fringe-field section [20], respectively.

\begin{tabular}{lc}
\hline \hline Vane length & $3623 \mathrm{~mm}$ \\
Number of cells & $317+\mathrm{TC}, \mathrm{FFS}$ \\
Intervane voltage & $81.0 \mathrm{kV}$ \\
Maximum surface field & $30.7 \mathrm{MV} / \mathrm{m}(1.72$ Kilpatrick $)$ \\
Average bore radius $\left(r_{0}\right)$ & $3.49 \mathrm{~mm}$ \\
$\rho_{t} / r_{0}$ ratio & $0.75\left(\rho_{t}=2.62 \mathrm{~mm}\right)$ \\
$a_{\min }$ & $2.23 \mathrm{~mm}$ \\
$m_{\max }$ & 2.13 \\
$\phi_{s, \max }$ & -30.6 \\
\hline \hline
\end{tabular}

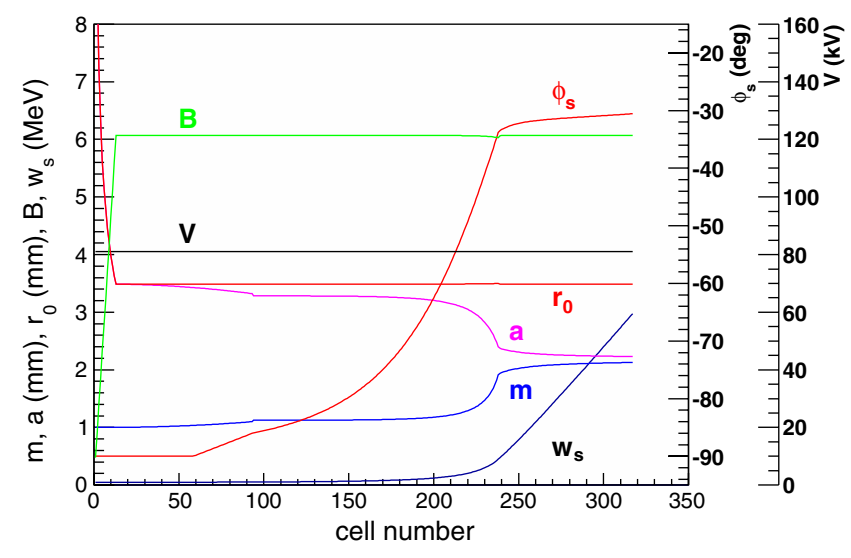

FIG. 1. Cell parameters of RFQ III designed with LINACSrfqDES. The $W_{s}$ means the energy of the synchronous particle.

larger than that of the two-term potential shape; therefore, the vane length can be shorter, and also the machining is easier. Since the J-PARC RFQ has suffered from sparking problems [7], the maximum surface field is set to be smaller than the typical value of 1.8 Kilpatrick. Figure 1 shows the obtained cell parameters as functions of cell number.

\section{SIMULATION}

The particle simulations were performed by using LINACSrfqSIM [11,12]. LINACSrfqSIM is a time-domain code to treat the space-charge effect correctly. In addition to the conventional multipole-expansion method (mpole mode), a multigrid Poisson solver is implemented in LINACSrfqSIM (Poisson mode). In the Poisson mode, the Poisson equation is solved with the boundary condition from the actual vane shapes to obtain both the external and space-charge fields. The Poisson solver calculates the electromagnetic field more accurately than the mpole method, especially, in the vicinity of the vane tips, and automatically correctly includes the image-charge effect. This feature is important for simulating high intensity RFQs.

In the simulation of RFQ III, the peak current of the input beam is $60 \mathrm{~mA}$, the normalized rms transverse emittance is $0.20 \pi \mathrm{mmmrad}$, the transverse distribution is water bag, input energy is $50 \mathrm{keV}$, and the phase distribution is uniform. The simulation results are presented in the next section.

\section{RESULTS}

In Table III, the simulation results are summarized. All of the following results are obtained using Poisson mode. The transmission is defined as a ratio of the accelerated particles.

Figure 2 shows emittance evolution as functions of cell number. The solid line represents the transverse emittance and the dashed line is the longitudinal one. The transverse 
TABLE III. Simulation results of J-PARC RFQ III.

Input beam current $60 \mathrm{~mA}$

Input transverse distribution $\quad 0.20 \pi \mathrm{mm}$ mrad, water bag

Input longitudinal distribution $50 \mathrm{keV}$ constant, uniform phase

Transmission

$98.5 \%$

Output transverse emittance

$0.21 \pi \mathrm{mm} \operatorname{mrad}$

Output longitudinal emittance

$0.11 \pi \mathrm{MeV} \operatorname{deg}$

emittance growth to the input emittance is $7 \%$; this is sufficiently small for the J-PARC purpose.

Next, the parameters concerning the equipartitioning are presented. In Fig. 3, the dotted line represents the ratio of the transverse beam radius to the longitudinal one, the dash-dotted line indicates the ratio of longitudinal phase advance to the transverse one. In the ACC, i.e., after cell 240, the longitudinal phase advance is getting weaker in comparison to the transverse one. Consequently, the longitudinal beam radius becomes larger relative to the

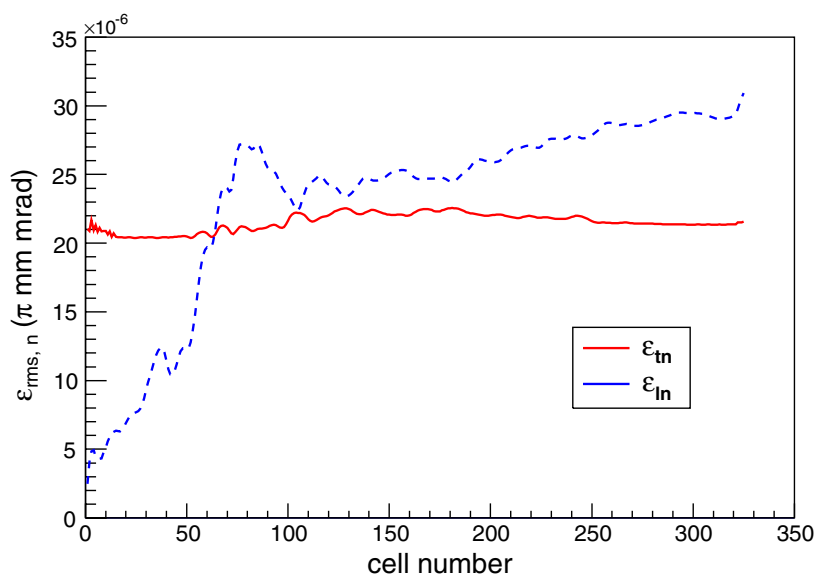

FIG. 2. Emittance evolution as functions of cell number. The solid line represents the transverse normalized rms emittance, and the dashed line is the longitudinal one.

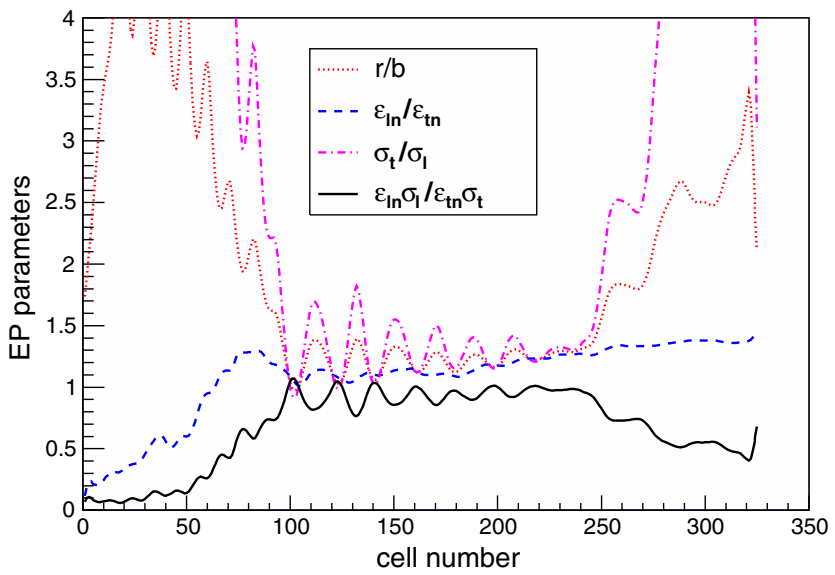

FIG. 3. Parameters related to the equipartitioning condition (EP parameters). When the equipartitioning condition holds, the solid line should be one. transverse one. The solid line is the equipartitioning ratio Eq. (9). It can be seen that $\varepsilon_{l n} \sigma_{l} / \varepsilon_{t n} \sigma_{t}=1$ in the GB, as the design aimed. The dashed line represents the ratio of longitudinal emittance to the transverse one; the emittance ratio is from 1.0 to 1.4 after the start point of the GB.

Figure 4 shows a Hofmann chart [21] for the emittance ratio of 1.2; the chart is specific to the emittance ratio. The horizontal axis of the Hofmann chart represents the ratio of the longitudinal phase advance to the transverse one (tune ratio), and the vertical axis is the ratio of the phase advance with space charge to that without space charge (tune depression). By plotting the trajectory of the tune depression as a function of the tune ratio on this plot, the status of

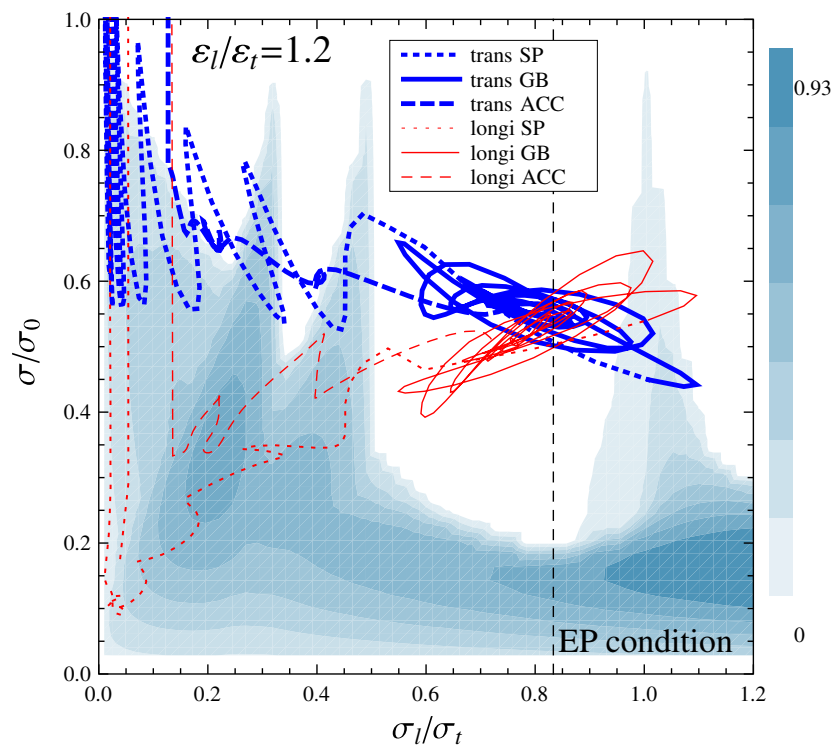

FIG. 4. Trajectories of the phase-advance ratio and depression of RFQ III on the Hofmann chart. The longitudinal/transverse emittances ratio of this Hofmann chart is 1.2.

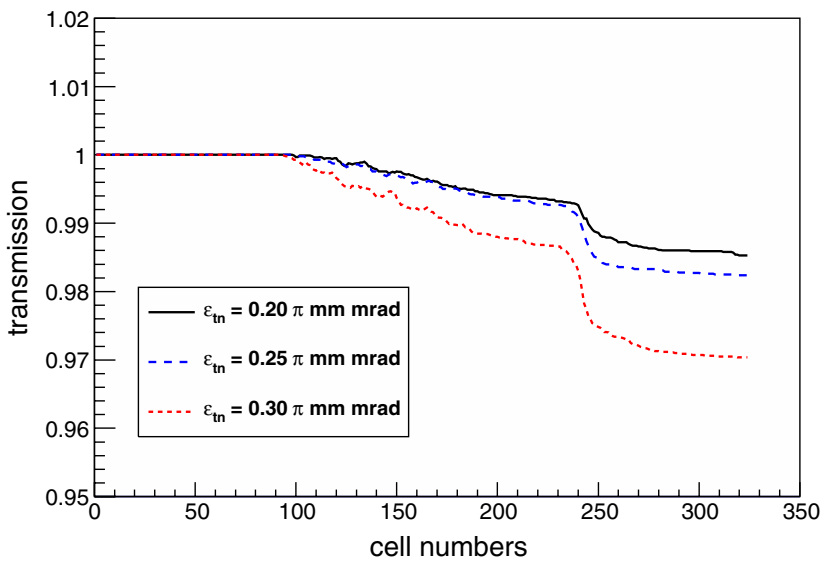

FIG. 5. Input-transverse-emittance dependence of the transmission. The solid, dashed, and dotted lines are transmissions for the input transverse emittances of $0.20,0.25$, and $0.30 \pi \mathrm{mm} \mathrm{mrad}$, respectively. 
TABLE IV. Input-transverse-emittance dependence of the RFQ III simulation.

\begin{tabular}{lccc}
\hline \hline Input emittance $(\pi$ mm mrad) & 0.20 & 0.25 & 0.30 \\
\hline Transmission $(\%)$ & 98.5 & 98.2 & 97.0 \\
Output transverse emittance $(\pi \mathrm{mm} \mathrm{mrad})$ & 0.21 & 0.25 & 0.29 \\
Output longitudinal emittance $(\pi \mathrm{MeV} \mathrm{deg})$ & 0.11 & 0.12 & 0.13 \\
\hline \hline
\end{tabular}

equipartitioning achievement and resonance crossing can be checked. In Fig. 4, the thick and thin lines represent the transverse and longitudinal trajectories of RFQ III in this space, respectively. The equipartitioning (EP) condition shows where Eq. (9) holds. In the GB, the trajectories are around the EP condition, where there is no energy to drive resonances, therefore emittance growth is small. The residual emittance growth is a consequence of some involvement with the $\sigma_{l} / \sigma_{t}=1$ resonance, and evolvement of the form factor $f$, which could also be mostly eliminated by further iteration of the design. In the ACC, the tune ratio is getting smaller and the longitudinal tune depression becomes smaller than the desirable value, i.e., the EP condition. However, the trajectories quickly pass through the resonance regions; therefore, no significant emittance growth occurs, as shown in Fig. 2. Lack of equipartitioning is not a serious threat for emittance growth in the design provided that the trajectories in the Hofmann chart do not dwell on a resonance.

Finally, the dependence of the transmission on the input emittance is shown in Fig. 5 and the transmissions and emittances are summarized in Table IV.

The solid, dashed, and dotted lines represent transmissions for the input transverse emittances of $0.20,0.25$, and $0.30 \pi \mathrm{mm}$ mrad, respectively. Figure 5 indicates that there is a bottleneck around the cell 240; this is because the constant-transverse-acceptance condition is started here, the start of the ACC. As shown in Table IV, the output emittances for the input emittances of 0.25 and $0.30 \pi \mathrm{mm}$ mrad cases are equal and smaller than the input ones. The reason is that some particles outside the constant transverse acceptance in the ACC are scraped, thus, the emittances are reduced. This bottleneck is unavoidable in conventional constant- $r_{0}$ design. However, in the design of RFQ III, sufficient transmission is obtained even when the input transverse emittance is $0.3 \pi \mathrm{mm}$ mrad. In addition, there is a strong requirement from the following linac for a transverse input emittance $0.3 \pi \mathrm{mm} \mathrm{mrad}$, so the scraping design provides good insurance for this requirement.

\section{CONCLUSION}

We performed the beam-dynamics design of an RFQ (RFQ III) for the beam-current upgrade of the J-PARC linac. For the convenience of fabricating the cavity and based on our fabrication experience, we adopted a modified conventional design, that is, both the average-bore radius and intervane voltage are constant along the RFQ, but the beam is also held equipartitioned through the GB. For the beam-dynamics design, LINACSrfqDES was used and LINACSrfqSIM for the particle simulation. Under the condition of input beam current of $60 \mathrm{~mA}$ and transverse emittance of $0.20 \pi \mathrm{mm} \mathrm{mrad}$, the transmission of $98.5 \%$, output transverse emittance of $0.21 \pi \mathrm{mm}$ mrad, and longitudinal emittance of $0.11 \pi \mathrm{MeV}$ deg are obtained. The obtained design satisfies the requirements of J-PARC RFQ III.

\section{ACKNOWLEDGMENTS}

The authors would like to express appreciation to Professor I. Hofmann for providing the data of the Hofmann chart.

[1] H. Hotchi, in Proceedings of IPAC2011, San Sebastián, Spain (EPS-AG, Spain, 2011), pp. 6-10.

[2] T. Koseki, in Proceedings of IPAC2011 (Ref. [1]), pp. 2499-2501.

[3] K. Hasegawa, in Proceedings of the 2007 Particle Accelerator Conference, Albuquerque, New Mexico (IEEE, New York, 2007), pp. 2619-2623.

[4] A. Ueno and Y. Kondo, in Proceedings of the 20th International Linac Conference, Monterey, CA, 2000 (SLAC, Menlo Park, CA, 2000), pp. 545-547.

[5] The RFQ for initial-stage linac is called RFQ I, and an RFQ fabricated as a spare of RFQ I [6], because the sparking problem of RFQ I occurred [7], is called RFQ II.

[6] T. Morishita, Y. Kondo, K. Hasegawa, F. Naito, M. Yoshioka, H. Matsumoto, Y. Hori, H. Kawamata, Y. Saito, and H. Baba, in Proceedings of LINAC10, Tsukuba (2010), pp. 521-523.

[7] K. Hasegawa, T. Kobayashi, Y. Kondo, T. Morishita, H. Oguri, Y. Hori, C. Kubota, H. Matsumoto, F. Naito, and M. Yoshioka, in Proceedings of IPAC2010 (Kyoto, Japan, 2010), pp. 621-623.

[8] K.R. Crandall, R.H. Stokes, and T.P. Wangler, in Proceedings of LINAC79, Montauk, New York (1979), pp. 205-216.

[9] L. M. Young, in Proceedings of the Particle Accelerator Conference, Vancouver, BC, Canada, 1997 (IEEE, New York, 1997), pp. 2752-2754.

[10] M. Comunian, A. Pisent, E. Fagotti, and P. A. Posocco, in Proceedings of the 11th European Particle Accelerator Conference, Genoa, 2008 (EPS-AG, Genoa, Italy, 2008), pp. 3536-3538.

[11] R. A. Jameson, Technical Report No. ORNL/TM-2007/ 001, Oak Ridge National Laboratory, 2007.

[12] R.A. Jameson, LINACS Design and Simulation Framework, Technical Report KEK/J-PARC Seminar 2012.

[13] "Mathematica, Wolfram Research Inc." [http://www .wolfram.com].

[14] T.P. Wangler, Technical Report No. LA-8388, Los Alamos National Laboratory, 1980. 
[15] K. R. Crandall, Technical Report No. LA-9695-MS, Los Alamos National Laboratory, 1983.

[16] R. A. Jameson, IEEE Trans. Nucl. Sci. 28, 2408 (1981).

[17] This type of buncher is not recommended, but worked for the KEKRFQ [18], RFQ I, RFQ II, and many early RFQs so was adopted.

[18] A. Ueno and Y. Yamazaki, in Proceedings of LINAC90, Albuquerque, New Mexico (1990), pp. 329-331.
[19] K. R. Crandall, in Proceedings of the 17th International Linear Accelerator Conference (LINAC94), Tsukuba, Japan, 1994 (KEK, Japan, 1994), pp. 227-229.

[20] K. R. Crandall, in Proceedings of LINAC84, Seeheim, Germany (1984), pp. 109-111.

[21] I. Hofmann, G. Franchetti, O. Boine-Frankenheim, J. Qiang, and R. D. Ryne, Phys. Rev. ST Accel. Beams 6, 024202 (2003). 